an estimated 43 million people. The Next Generation Cassava Project is implementing genomic selection to systematically improve the yield and nutritional value of this African staple. As modern techniques are brought to bear on other crops, such as yams and millets, they will continue to enrich the world's table.
COMPETING FINANCIAL INTERESTS

The author declares no competing financial interests.

1. International Crops Research Institute for the SemiArid Tropics. The World Sorghum and Millet Economies: Facts, Trends and Outlook. (ICRISAT, 1996).

2. Varshney, R.K. et al. Nat. Biotechnol. 35, 969-976 (2017).

3. Voss-Fels, K. \& Snowdon, R.J. Plant Biotechnol. J. 14, 1086-1094 (2016).
4. Witek, K. et al. Nat. Biotechnol. 34, 656-660 (2016).

5. New Partnership for Africa's Development (NEPAD) Comprehensive Africa Agriculture Development Programme. (NEPAD, 2003).

6. CYMMIT. Drought Tolerant Maize for Africa Seed Scaling. http://www.cimmyt.org/project-profile/ drought-tolerant-maize-for-africa-seed-scaling-dtmass/ (CYMMIT, 2015).

\title{
Mosaic mice ace functional genomics
}

Understanding the function of a gene ultimately requires experiments in the native tissue environment where it is expressed. In mammals, however, the technologies available for such studies are often limited. Writing in Cell, Benedito and colleagues ${ }^{1}$ present ifgMosaic, a new set of tools that finally allows mouse geneticists to efficiently create the kinds of genetic mosaics that have long been indispensable to fly researchers. "This fills a real gap. We need to be able to do more clonal analysis in mice," says Bon-Kyoung Koo, a stem cell biologist at the Institute of Molecular Biotechnology in Vienna.

In a mosaic animal, different sets of cells carry different genetic material. This is an ideal system for analyzing gene function since the phenotypes of various cell populations can be compared in the same tissue without other confounding factors. In flies, mosaics are readily created by mitotic recombination, but developing similar techniques for mice has proved challenging. Previous methods have been hampered by a low percentage of modified cell clones or unreliable coexpression of reporter genes.

The method of Benedito and colleagues ${ }^{1}$ exploits the mutually exclusive loxP sites used for combinatorial labeling of neurons in the Brainbow or Confetti mice popular in neuroscience. Their standard constructs consist of an array of fluorescent markers separated by three different loxP sites. Recombination by tamoxifen-induced Cre recombinase has only three possible outcomes, each leading to exclusive expression of one of the fluorescent markers. ifgMosaic constructs come in two flavors, allowing for easy analysis of cell numbers and morphology: iMb-Mosaic, with membranebound fluorescent proteins, and iChr-Mosaic, with chromatin-targeting fluorescent proteins. Each marker can be coupled to the expression of a gene of interest using a $2 \mathrm{~A}$ peptide for bicistronic expression of marker and gene. $\mathrm{iMb}$ and iChr constructs can be combined to study the consequences of combinatorial expression of up to six genes, creating 15 different subpopulations (in addition to unrecombined wild-type cells) that express either one or a combination of two genes in the constructs.

"This strategy is very elegant and takes the basic idea used in the Confetti mice to the next level," says Janet Rossant, a developmental

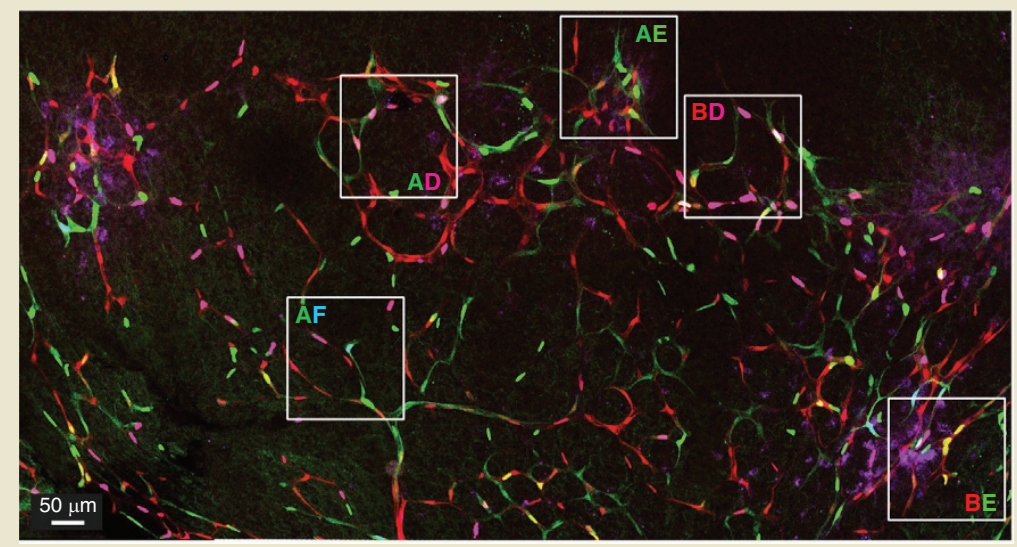

Image taken from Ref. 1

biologist at the Hospital for Sick Children in Toronto. "The combination of nuclear and membrane labels is especially important for downstream analyses."

Generating dual ifgMosaic animals does require the integration of a single copy of large multi-gene constructs into the genome-itself not a small feat. To facilitate this process, the authors offer two integration methods. In the first, fertilized eggs are injected with a bacterial artificial chromosome (BAC) containing part of the Rosa26 locus and isolators to minimize the effects of neighboring regulatory elements and ensure efficient transcription. Random genomic insertion of the $\mathrm{BAC}$ is mediated by flanking piggyBac transposon inverted repeats and a co-injected piggyBac transposase. Up to $26 \%$ of the resulting pups carry a genomic BAC insertion. Cloning of the expression cassettes with the genes of interest can be done by recombineering in Escherichia coli.

Although these BAC constructs worked well in transgenic mice, they performed less well in cell culture. The authors therefore also developed a system to target the ifgMosaic constructs to the endogenous mouse Rosa26 locus. First, they generated a mouse embryonic stem cell line with a modified Rosa26 locus containing all the constant elements of the ifgMosaic constructs. The specific elements were then inserted into a unique sequence at the center of the modified locus using CRISPR-Cas9, achieving a $56 \%$ integration rate.

"The authors make all these constructs freely available," says Koo. "This will be an enormous help when trying to set up the system, although assembling the necessary constructs for one's own experiments will not be entirely straightforward for some labs. One also has to keep in mind that by its very nature, mosaic analysis can be misleading if the gene of interest works in a non-cell-autonomous manner."

How the constructs can be used is highlighted by the authors' analysis of the role of Notch and VEGF pathways in neurogenesis and angiogenesis in mouse embryos. For example, they express combinations of Notch and VEGF signaling activators and inhibitors, and analyze how clones expressing different combinations proliferate in the same tissue. Endothelial cells with high Notch activity do not benefit from additional VEGF signaling, whereas cells with low VEGF activity proliferate only if Notch activity is also low.

Many extensions of ifgMosaic can be envisioned. "It will be essential to further develop the method to enable gene inactivation, which can probably be done by expressing an shRNA [short hairpin RNA], an sgRNA [single guide RNA] in combination with Cas9, or a dominant negative," says Rossant. "This method can potentially provide such a wealth of information that data analysis will be the next challenge."

Markus Elsner, Senior Editor

1. Pontes-Quero, S. et al. Cell 170, 800-814 (2017). 